\title{
Characterization and System Identification of XY Flexural Mechanism using Double Parallelogram Manipulator for High Precision Scanning
}

\author{
Mahesh S. Shewale ${ }^{1}$, Ali Razban ${ }^{1}$, Suhas P. Deshmukh², \\ Sharad S. Mulik ${ }^{3}$, Abhishek D. Patange ${ }^{3}$ \\ ${ }^{1}$ Department of Mechanical Engineering, Purdue School of Engineering \& Technology, \\ IUPUI, Indianapolis IN 46202 \\ ${ }^{2}$ Department of Mechanical Engineering, Govt. College of Engineering, Karad, \\ Dist. Satara (MH), India \\ ${ }^{3}$ Department of Mechanical Engineering, RMD Sinhgad School of Enginering, \\ Pune (MH), India
}

\begin{abstract}
This article represents modeling of double parallelogram flexural manipulator derived from basic classical mechanics theory. Fourth order vibration wave equation is used for mathematical modeling and its performance is determined for step input and sinusoidal forced input. Static characterization of DFM is carried out to determine stiffness and force deflection characteristics over the entire motion range and dynamic characteristics is carried out using Transient response and Frequency response. Transient response is determined using step input to DFM which gives system properties such as damping, rise time and settling time. These parameters are then compared with theoretical model presented previously. Frequency response of DFM system gives characteristics of system with different frequency inputs which is used for experimental modeling of DFM device. Here, Voice Coil Motor is used as Actuator and optical encoder is used for positioning sensing of motion stage. It is noted that theoretical model is having 5\% accuracy with experimental results. To achieve better position and accuracy, PID and LQR (Linear Quadratic Regulator) implementation was carried out on experimental model. PID gains are optimally tuned by using Ziegler Nichols approach. PID control is implemented experimentally using dSPACE DS1104 microcontroller and Control Desk software. Experimentally, it is observed that positioning accuracy is less than 5 micron. Further multiple DFM blocks are arranged for developing $\mathrm{XY}$ flexural mechanism and static characterization was carried out on it. The comparison of experimental and FEA results for X-direction and Y-direction is presented at end of paper.
\end{abstract}

Keywords: Flexures, PID Control, System Identification, dSPACE, Precision Scanning, DS1 104, Voice Coil Actuator

This is the author's manuscript of the article published in final edited form as:

Shewale, M. S., Razban, A., Deshmukh, S. P., Mulik, S. S., \& Patange, A. D. (2020). Characterization and System Identification of XY Flexural Mechanism Using Double Parallelogram Manipulator for High Precision Scanning. In A. Kumar \& S. Mozar (Eds.), ICCCE 2019 (pp. 387-404). Springer. https://doi.org/10.1007/978-981-13-8715-9_47 


\section{Introduction}

The demand for high precision positioning has been increased rapidly with the advancement in the domain of mechatronics, control systems and its integration with mechanical systems [1-3]. Nano-positioning stages are widely used in various applications such as biomedical, stereo-lithography for development of prototypes, laser scanning, micromachining and scanning probe microscopy etc. [4-6]. Hence, the use of accurate and precise instruments has almost become inevitable that eventually lead to research work related up to submicron level accuracy and resolution [5-7]. Different XY scanning mechanisms are under development but have several constrains such as limited range of scanning, restricted performance in sense of accuracy, backlash, fixed degrees of freedom, reliance of motion on one another etc. Moreover the main aspect is to design a proper control system and interface it to provide precise control for desirable working [8-10]. In the recent years, more focus has been given to compliant or flexural mechanisms for improving the performance of such devices [11-13]. Flexures are nothing but the bending members which deforms in a particular direction on the application of load. Flexures are more suitable due their distributed flexibility in providing the desired motion in the required direction along with the advantage of absence of development of assembly; no wear/tear hence no need of greasing/oiling and exclusion of backlash [14].

Present work is one of the attempts to design, manufacture, system identification, integration and PID control implementation on basic building block of flexural mechanism i.e. Double Flexural Manipulator (DFM) [15]. Section 1 gives comparative study of fundamentals of flexural mechanisms and applications. Section 2 explains proposed mechanism, its manufacturing, assembly and monolithic structure of XY mechanism (presented in Awatar's Thesis) which uses DFM as building block. Section 3 presents PID control implementation on proposed system. It outlines system integration (i.e. interfacing of sensor and actuator to PC via dSPACE DS1104 microcontroller. Further this section explains system identification and comparison of predicted model and experimental results. Section 4 presents the tuning of system using PID control and application on DFM and design and implementation of LQR control strategy. Section 5 presents development of XY flexural mechanism and its static characterization. Section 6 gives concluding remarks and future scope of the work.

\section{Review of Flexural Mechanisms}

The flexural mechanism yields a relative motion between the rigid links and connection between these rigid links decides a path of motion [16-19]. These connections in case of rigid links are pin joints, roller joints, ball bearings etc. But conventional joints have a friction and backlash during motion and degrade the quality of motion (i.e. smoothness, repeatability, positioning accuracy etc.) [20-22]. Hence new era of flexural mechanism is invented to overcome these difficulties. These flexural mechanisms generate a motion between rigid links via flexible joints. In general, flexural mechanisms classified into two types based on joints used in 
mechanism. Firstly one domain rely on mechanism with flexural hinges (where compliance is at single point), and another domain uses flexible planar joints (where compliance is distributed over the entire joint) such as flexible plates and beams to achieve desired pattern of motion. Flexural mechanisms with hinges typically used for rotational type of motion and planar type joints are used for linear type of motion [23 24]. Different types of mechanisms and its building blocks are outlined as below.

Fig.1 shows a flexural hinge (single axis and multi-axis) and mechanisms developed. It also illustrates 3 degrees of freedom tripod mechanism, 2 degrees of freedom motion stage, and positioning stage with six axes. Second domain of mechanism uses flexible plates, beams and its combinations (see Fig.2) as building block. Compared to Flexural hinges, Flexible planar joints have distributed compliance which is more suitable where linear motion is needed. Fig.2 shows flexible planar joints and mechanisms developed. Different flexible planar joints are designed and developed, basically these developments mainly concentrates on reduction or to achieve a zero parasitic motion and rotation of motion stage for linear scanning.

Finite Element Analysis is performed to compare the performance parameters (such as parasitic motion, rotation of motion stage and stiffness of mechanism in orthogonal directions) using ANSYS FEA tool. It is identified that double parallelogram flexural manipulator exhibits zero parasitic error motion theoretically and little amount of rotation. Theoretical and FEA simulations shows cantilever beam has a large parasitic error motion and DFM shows zero parasitic error motion. DFM gives zero parasitic error motion but gives a small rotation of motion stage. DFM is best suitable flexible planar joint for linear scanning type mechanism. Hence, experimental setup for DFM is developed and static and dynamic characteristics have been determined using experimental identification. Next section discusses the DFM experimental setup and its system integration with PC via dSPACE DS1104 microcontroller.

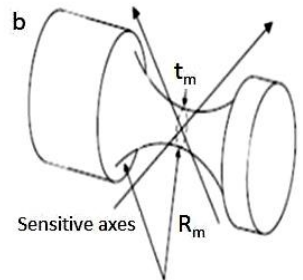

Multiple Hinge

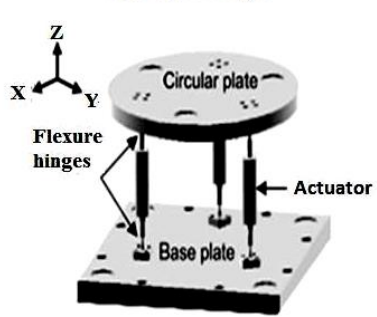

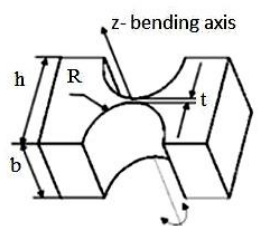

Single Axis Hinge

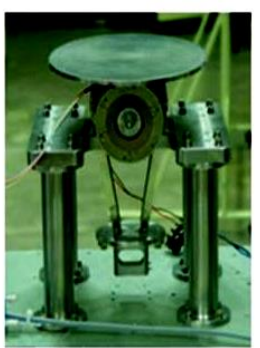

Figure 1. Various flexural mechanisms. 


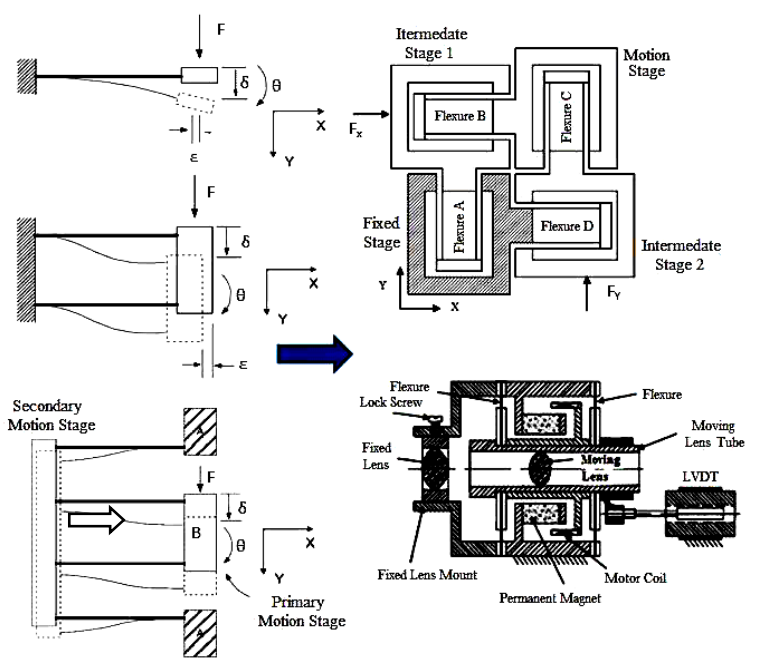

Figure 2. Mechanisms developed using planar flexible joints

\section{Experimental Setup: DFM}

Experimental setup for DFM testing, characterization, control implementation is developed. It consists of DFM, sensor (optical encoder with $50 \mu \mathrm{m}$ positioning resolution from Renishaw Inc.), Actuator (voice coil motor by BEI-Kimco Inc.), dSPACE DS1104 microcontroller, Linear Current Amplifier, and PC. Fig. 3 shows DFM with sensor and actuator mounted on it [25-26]. Fig.4 \& fig.5 shows DFM system integration with PC via dSPACE DS 1104 microcontroller. Fig.5 shows entire setup mounted on optical table. Optical table is used for ground vibration isolations. PC consists of MATLAB, Simulink and Control Desk software which runs in synchronization with each other and collects the data from sensor and generates a signal to actuator according to logic created in SIMULINK model.

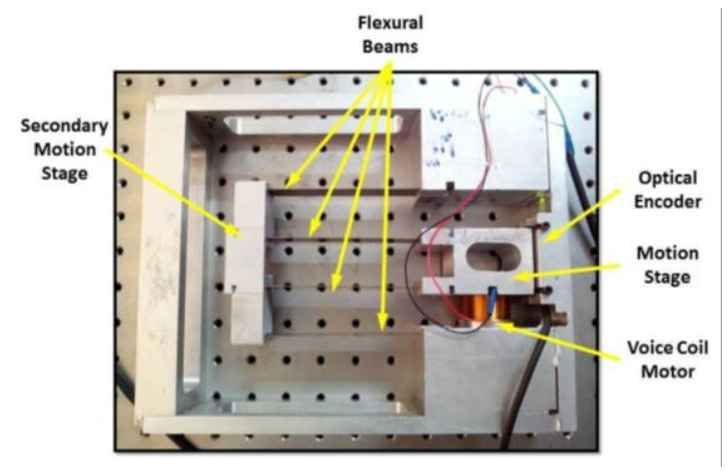

Figure 3. Manufactured Double Flexural Manipulator (DFM) 


\section{A. Evaluation of Stiffness:}

Stiffness is a system property to be determined at very low speed on operation which is typically less than the three times of its natural frequency. An experimental stiffness characteristic (extension and retraction motion) of DFM Mechanism is shown in graph of deflection Vs Force in fig 6. An experimental result shows forward path motion and backward path motion having close match. Table 1 shows experimental results i.e. deflection and stiffness; it is observed error between theoretical results and experiments within less than $1 \%$.

Table 1.Observation Table and Calculations

\begin{tabular}{|c|c|c|c|c|c|}
\hline \multirow[t]{2}{*}{ Load } & \multicolumn{2}{|c|}{ Deflection } & \multicolumn{2}{|c|}{ Stiffness } & \multirow{2}{*}{$\begin{array}{c}\% \\
\text { Error }\end{array}$} \\
\hline & Th. & Pr. & Th. & Pr. & \\
\hline-5 & -7.692 & -7.73 & 0.65 & 0.6468 & 0.49 \\
\hline-2.5 & -3.846 & -3.874 & 0.65 & 0.6453 & 0.724 \\
\hline 2.5 & 3.846 & 3.877 & 0.65 & 0.6448 & 0.802 \\
\hline 5 & 7.692 & 7.711 & 0.65 & 0.6484 & 0.243 \\
\hline
\end{tabular}

\section{B. Evaluation of Damping Factor:}

Transient force is applied to give initial displacement is given to motion stage and then permitted to pulsate without restrictions up till it arises to end; that is to achieve a Transient Response. Experimental results are obtained and graph of deflection vs time is plotted as shown in fig.7 and transient response in fig.8. The damping coefficient is determined from Logarithmic decrement. Eq.(1) for Logarithmic decrement is stated as,

$$
\delta=\frac{1}{n}\left[\log \left(\frac{X_{0}}{X_{n}}\right)\right]
$$

Eq.(2) for damping factor is given by,

$$
\xi=\frac{\delta}{\sqrt{4 \pi^{2}-\delta^{2}}}
$$

The value of Logarithmic decrement was observed as 0.218308 and damping factor as 0.034785 experimentally. 


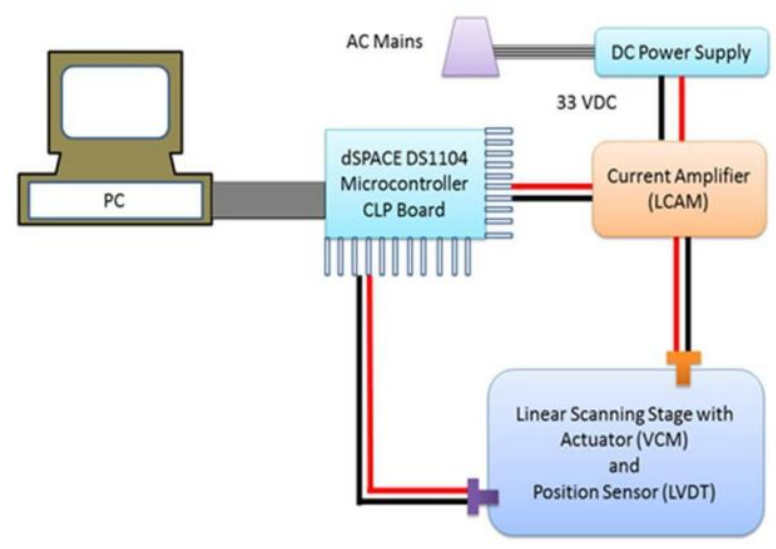

Figure 4. Mechatronic integration of proposed system

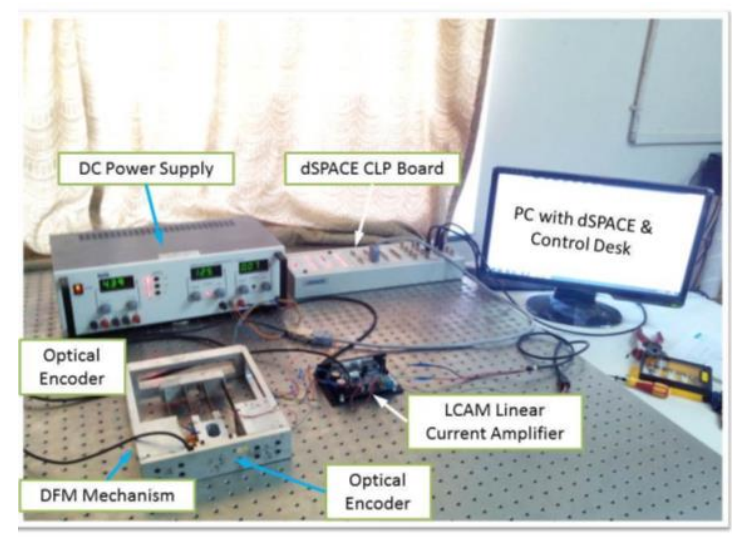

Figure 5. Development of Experimental Setup of proposed system

\section{Identification of the System}

To develop transfer function experimentally it necessitates achieving the system identification for the proposed system. The ratio of actuator input signal to location of motion stage with constant amplitude and variable frequency will give transfer function of the system. Initial conditions are assumed to be zero. The frequency response is obtained by providing input voltage in sinusoidal form and monitoring resultant output positions. For real time monitoring of frequency response, an algorithm is designed in MATLAB Simulink. A frequency response curve as shown in fig. 7 is used to investigate the natural frequency and the phase change with 0.08 volts amplitude as input and 1 to $70 \mathrm{~Hz}$ frequency range. The highest frequency of $24.51 \mathrm{rad} \mathrm{s}-1$ is identified. 
Here, though we are finding movements in $\mathrm{X}$ and $\mathrm{Y}$ both directions, we are concerned about double flexural mechanism which is having single degree of freedom (DOF). Therefore only one input output transfer function of is estimated as Eq.(3) below,

$\boldsymbol{G}(\boldsymbol{s})=\frac{1}{s^{2}+1.705 s+600.7}$

Fig. 8 shows comparison of experimental and model results of DFM. It shows close matching with each other. Next section onward PID Control design and implementation on DFM is discussed in details.

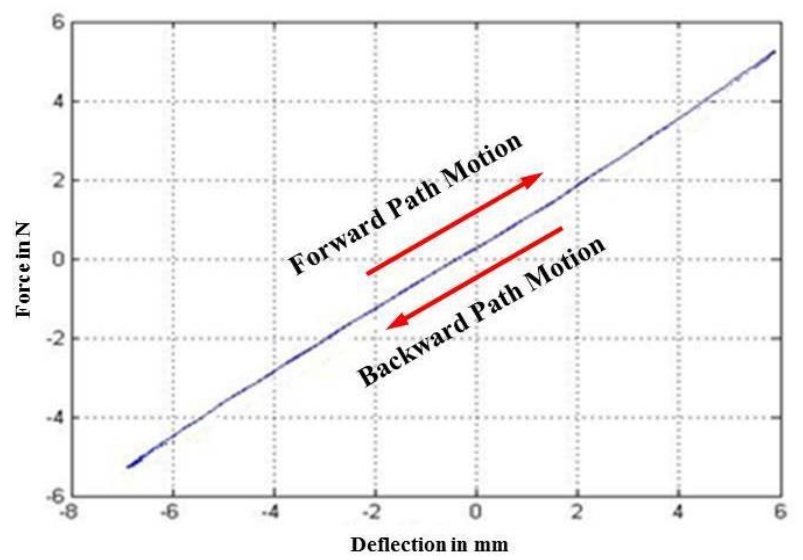

Figure 6. Stiffness Characteristics of DFM

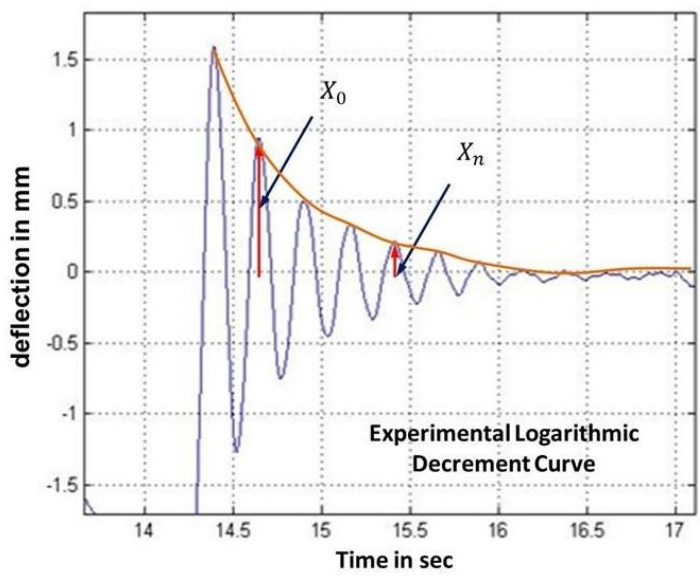

Figure 7. Logarithmic decrement curve 


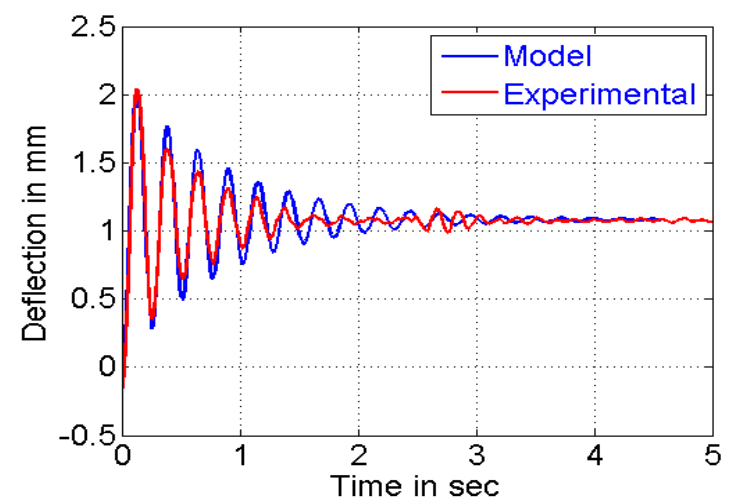

Figure 8. Comparison of Transient Response for Experimental and Model

\section{PID Control Implementation}

For achieve control of motion stage position in high precision applications the design and implementation of PID controller is carried out on proposed system. PID control consists of three elements proportional element, integral element and derivative element. PID takes care of error in all three tenses (i.e. present, past and future). Tuning of constant parameters gives precise control of position of motion stage. The control equation for PID control system is shown as Eq.(4) below,

$\boldsymbol{u}(\boldsymbol{t})=K_{p} e(t)+K_{i} \int e(t) d t+K_{d} \frac{d e(t)}{d t}$

Where, ${ }_{p}$ : Proportional, $K_{i:}$ : Integral and $K_{d: \text { derivative gains }}$

$$
e(t)=\text { Error and } \mathrm{u}(\mathrm{t}) \text { is controller output. }
$$

Ziegler-Nichols approach is adopted for optimal tuning of PID gains. As in the mentioned above, the $\mathrm{K}_{\mathrm{i}}$ and $\mathrm{K}_{\mathrm{d}}$ gains are set to zero. The output of the loop starts to oscillate when proportional gain reaches the ultimate gain $\mathrm{Ku}$ with gradual increment of proportional gain. To set the gains, ultimate gain $\mathrm{K}_{\mathrm{u}}$ and oscillation period $\mathrm{Pu}$ are used. Tuned PID parameters are applied in real time for precise control of position of DFM motion stage. Fig.9 shows a real-time control of position motion stage and shows a comparison between reference signal and actual signal. Fig.10 shows error between reference and actual position.

PID control strategy further can be used for precise control of position of motion stage. Further LQR is also designed and implemented on DFM and its performance is compared with PID control strategy. 


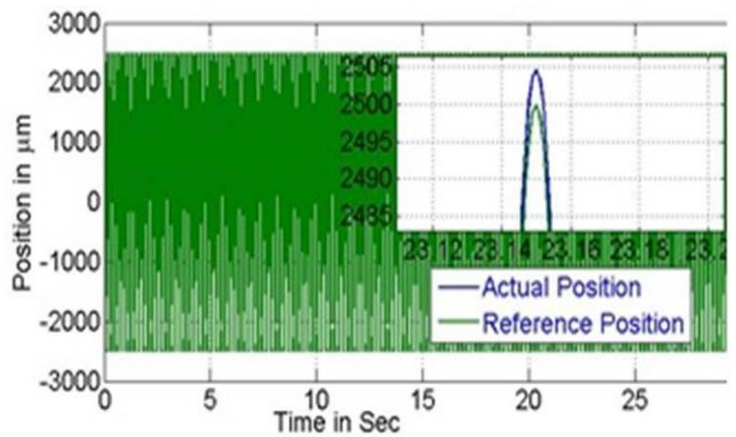

Figure 9. Comparison of actual and reference position

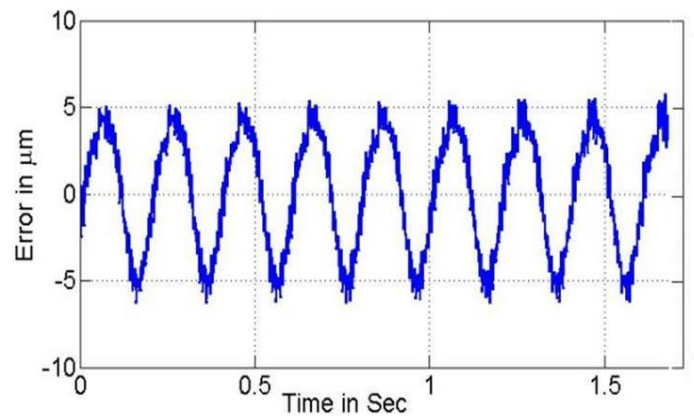

Figure 10. Actual error signal observed

\section{LQR Implementation}

The LQR was used on the setup. Full state feedback is central to the LQR designs. Therefore, we use the Kalman Filter to estimate state variables. The aim of the standard LQR problem is to converge the state trajectories to zero in an optimal way.

Choosing $q_{1}, q_{1}, q_{2}, q_{2}$ as the state variables, the equations can be written in the form: Where

$$
\boldsymbol{x}=\left[\begin{array}{l}
q_{1} \\
\dot{q}_{1} \\
q_{2} \\
\dot{q}_{2}
\end{array}\right]
$$

and $\mathrm{A}, \mathrm{B}, \mathrm{C}$ matrices are given by 


$$
\begin{aligned}
\mathbf{A}= & {\left[\begin{array}{cccc}
0 & -1.07 e 4 & 0 & -5.8 e 6 \\
1 & 0 & 0 & 0 \\
0 & 1 & 0 & 0 \\
0 & 0 & 1 & 0
\end{array}\right], \mathbf{B}=\left[\begin{array}{l}
1 \\
0 \\
0 \\
0
\end{array}\right] } \\
& \text { and } \mathbf{C}=\left[\begin{array}{llll}
0 & 58.67 & 0 & 5.55 e 5
\end{array}\right]
\end{aligned}
$$

The general criterion used to arrive at the control law is in Eq.(5)

$$
I=\int_{t_{0}}^{t_{f}}\left[x^{\prime}(t) Q x(t)+u(t)^{\prime} R u(t)\right] d t+\frac{1}{2} x^{\prime}\left(t_{f}\right) P_{f} x\left(t_{f}\right)
$$

Subject to

$$
Q \geq 0, R>0, P_{f} \geq 0, x\left(t_{0}\right)=x 0,\{A, B\}
$$

and

$$
\dot{x}=A x B u
$$

The deviation of $\mathrm{x}$ from the desired trajectory is penalized quadratically with a symmetric positive semi definite weighting matrix Q. Also, the input $u$ is quadratically weighted with positive definite (symmetric) matrix R in order to keep all inputs within the range of the particular actuator. The pf term allows the designer to specifically penalize the state trajectory at the final time. The solution of the above problem can be derived to be:

$$
u(t)=-R^{-1} B^{\prime} P(t) x(t)=-L(t) x(t)
$$

Where,

$$
\begin{gathered}
L(t)=R^{-1} B^{\prime} P(t) \\
-\dot{\boldsymbol{P}}(t)=P(t) A+A^{\prime} P(t)-P(t) B R^{-1} B^{\prime} P(t)+Q
\end{gathered}
$$

With the boundary condition

$$
P(t)=P_{f}
$$

Suppose the designer wishes the system to attain a desired state $\mathrm{x}$ ref. The above control law will then have to be modified.

Define a new state variable:

$$
r=x_{r e f}-x
$$

The system equations take the form:

$$
r^{*}=A r+B u-A x_{r e f}
$$

and

$$
\boldsymbol{y}=C\left(x_{r e f}-r\right)
$$

The optimal control input then becomes:

$$
\boldsymbol{u}=+L r-L x_{r e f}
$$


Hence

$\dot{\boldsymbol{r}}=A r-B L r+B L x_{r e f}-A x_{r e f}$

To represent this new system in the standard form, we want equations of the form

$$
\dot{\boldsymbol{r}}=A r+B u
$$

With the input of the form

$$
\boldsymbol{u}=-L r+v *
$$

Comparing,

$$
B v=\text { BLxref }- \text { Axref }
$$

Also, since LQR control will take $\mathrm{x}$ to zero, the new system output $\mathrm{y}$ will approach

$$
y_{r e f}=C x_{r e f}
$$

As seen in the curve (see Fig.11), there is a distinct steady state error. This is partly because we generated our reference input based on model information which is subject to error. Also, 'zero shifting' due to existence of fields, presence of stiffness in the beams at the $\mathrm{y}=0$ might have caused this steady state error. The choice of weighting matrices is extremely important. If you choose a $\mathrm{Q}$ that gives more importance to the first state variable, you'll see visible oscillations in position after settling. Notice the shape of the curve in the fig.12. It can be clearly seen that the position curve closely follows the shape of the amplified and shifted disturbance curve.

DFM gives better control performance for linear scanning and accuracy of position of motion stage can be further improved by tightly tuning PID control parameters. DFM can be further used for development of XY flexural mechanism to achieve an orthogonal linear scanning and has numerous applications in precision scanning. Shourya Awtar developed family of such mechanisms and we have used one of the mechanisms for further investigation [1]. Next section discusses about XY flexural mechanism and its static characterization. 


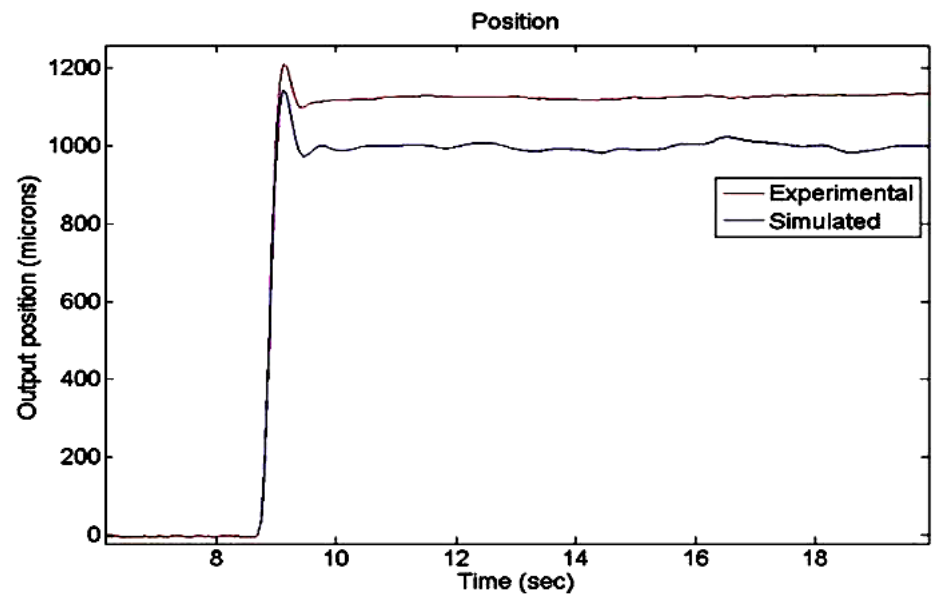

Figure 11. Position plot for regulation using LQR

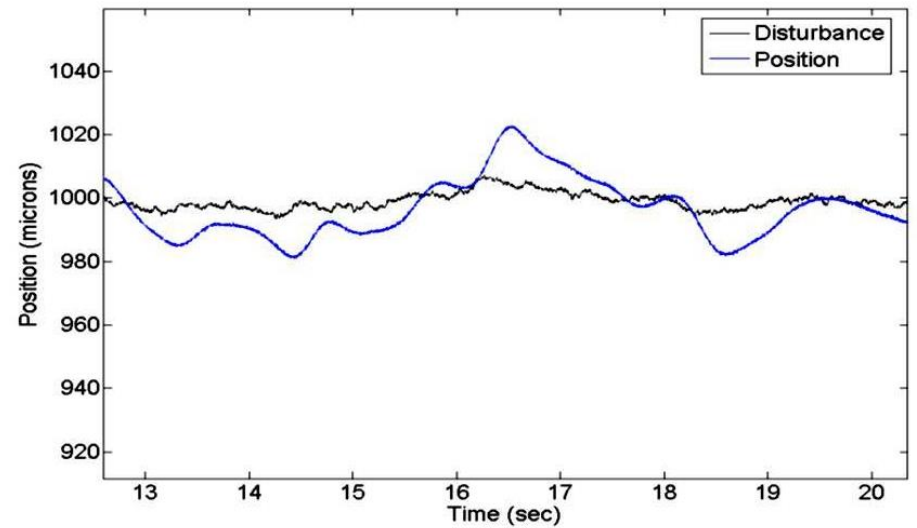

Figure 12. Simulated response plotted against generated disturbance (LQR)

\section{Development of XY Flexural Mechanism}

Multiple DFM blocks are arranged such that XY scanning is achieved. Fig.13 shows a $\mathrm{XY}$ mechanism manufactured for $\mathrm{XY}$ for precision scanning.

Fig.14 shows a block diagram representation of experimental setup, it consists of sensors (dial gauges), it's mounting and loading platform. Fig.15 shows an arrangement of mounting of XY flexural mechanism on optical table and alignment of dial gauges for recording $\mathrm{X}$ and $\mathrm{Y}$ direction motions. 


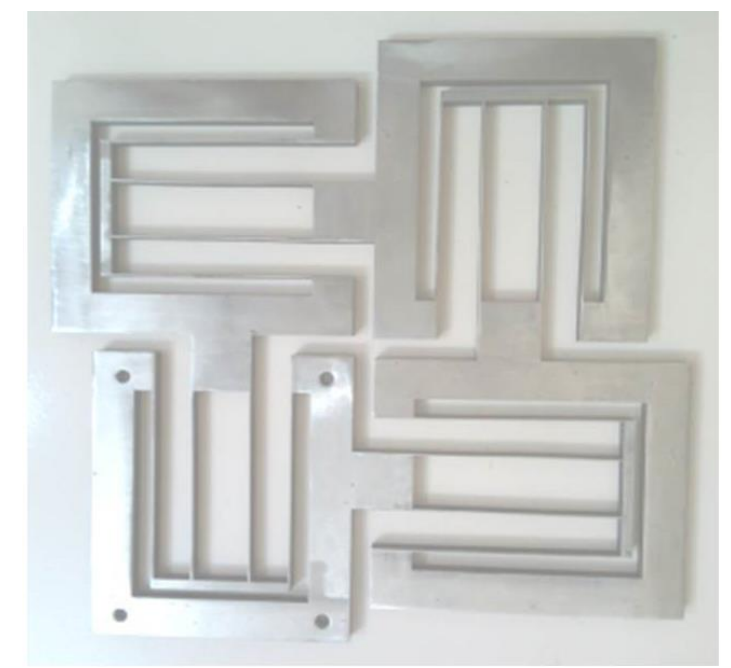

Figure 13. Developed XY Flexural Mechanism

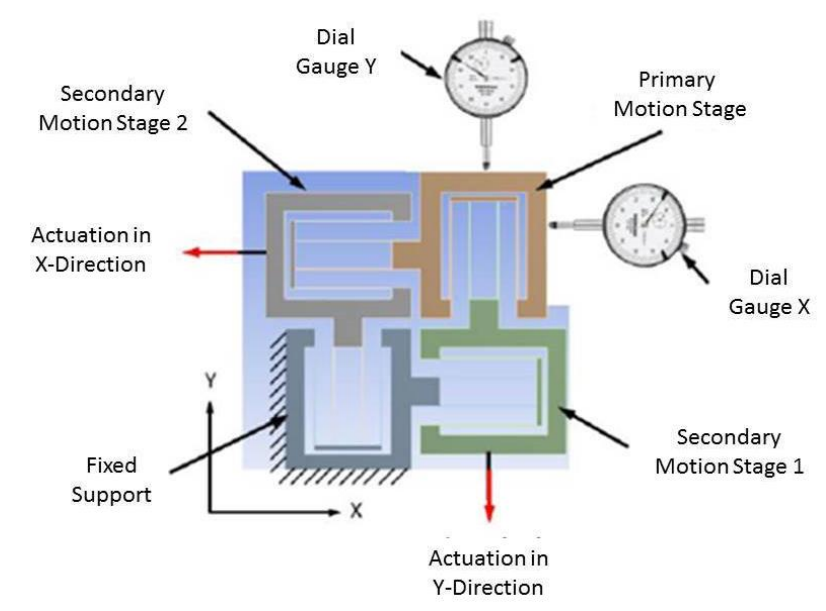

Figure 14. Layout of experimental setup

Fig.15 illustrates an experimental setup with two dial gauges which measures displacement of motion stage in $\mathrm{X} \& \mathrm{Y}$ directions respectively. These dial gauges have a resolution of $10 \mathrm{~m}$ and range of measurement is $25 \mathrm{~mm}$ maximum. Red color flexible wires are tied at actuator location to provide an appropriate actuation in $\mathrm{X} \&$ Y directions. Load is applied using weight pan with an increment of 25 grams. For each increment of load deflection of motion stage is recorded. Load in weight pan (maximum $35 \mathrm{~N}$ i.e. $3.5 \mathrm{Kg}$ ) is given such that maximum of $7.5 \mathrm{~mm}$ displacement is achieved. 


\section{Static Characterization}

Fig. 16 \& fig. 17 shows comparison of experimental results and FEA results. It is noted that the finite element analysis results yields good matching with experimental results. During the actuation of $\mathrm{X}$-stage, $\mathrm{Y}$-stage deflection was recorded continuously by other dial gauge to record motion in X-stage. It is observed experimentally 25 micron motion in X-direction when actuation is given in Y-direction. Further, it is noted that motion is not due to parasitic or cross coupling but due to manufacturing error or surface finish. Similar, results are observed in other direction, hence, it can be concluded that zero parasitic motion in y-direction eliminated for the desired $\mathrm{x}$ direction motion and zero parasitic motion in $\mathrm{x}$-direction eliminated for the desired $\mathrm{y}$ direction motion. Slope of force deflection curve is a stiffness of flexural mechanism in the direction of actuation.

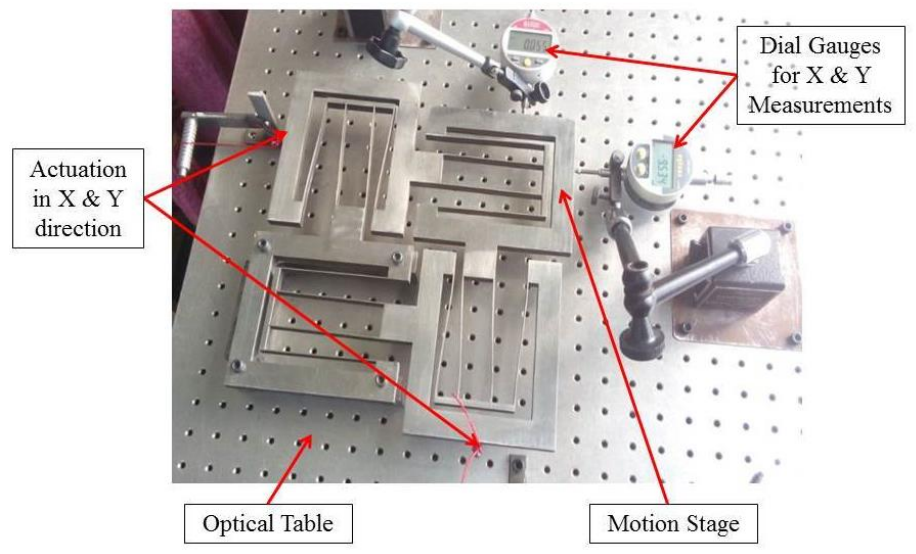

Figure 15. Developed of Experimental Setup 1

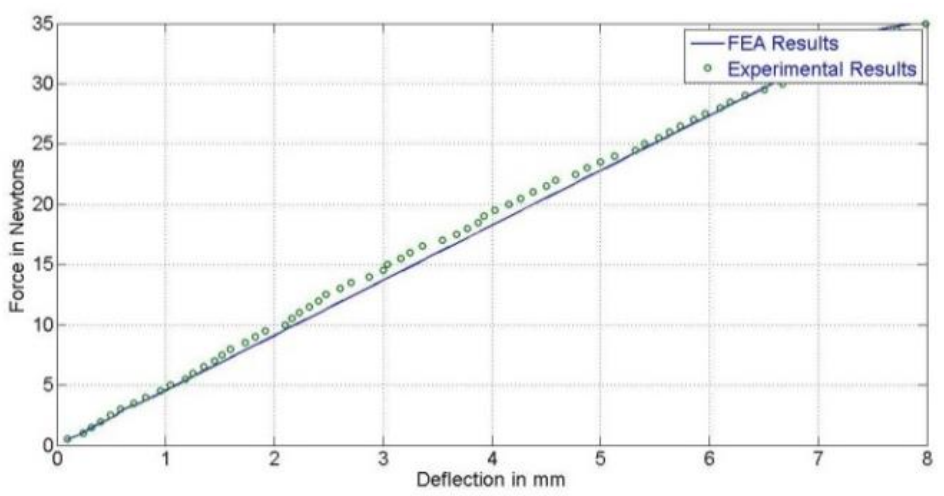

Figure 16. Comparison of experimental and FEA results for X-direction 


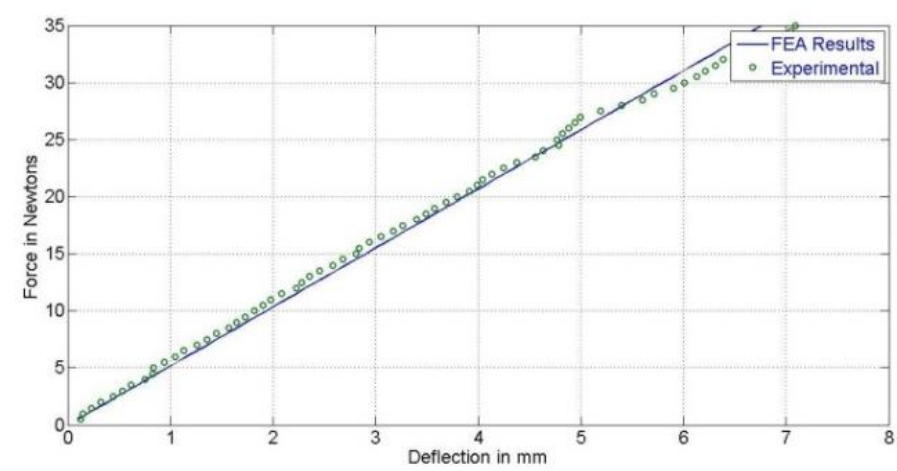

Figure 17. Comparison of experimental and FEA results for Y-direction

\section{Conclusion}

The modeling of double parallelogram flexural manipulator (DFM) is presented and further experimentally investigated. DFM is characterized in two different domains (1) Static characterization is carried out to determine force deflection characteristics over the entire motion range; (2) Dynamic characterization are carried out using Transient response and Frequency response. Transient response is determined using step input to DFM which gives system property damping. It is noted that theoretical model is having 5\% accuracy with experimental results. Frequency response plots for the mechanism denotes behaviour of system for different frequency inputs. This frequency response plot is utilized to model DFM device experimentally. Experimental model i.e. transfer function of DFM with input as analog voltage signal and output as displacement of motion stage is used to obtain frequency response using constrained minimization approach. Experimental model is then compared with analytical model developed using fourth order wave equation and it is seen that results of both the models have very good agreement. PID control implementation was done on developed experimental model. To achieve better position and accuracy, PID and LQR implementation was carried on developed experimental model. PID parameters (Proportional, Integral, Derivative gains) are tuned using Ziegler Nichols Method. PID control is applied using dSPACE DS1104 microcontroller and Control Desk software. Experimentally, it is observed that positioning accuracy is less than 5 microns. XY flexural mechanism was developed by arranging multiple DFM blocks and static characterization was carried out. It is observed 25 micron motion in $\mathrm{X}$ direction when actuation is given in $\mathrm{Y}$-direction experimentally. This motion is not due to parasitic or cross coupling but due to manufacturing error or surface finish. Hence we concluded that zero parasitic motion in y-direction eliminated for the desired $\mathrm{x}$-direction motion and zero parasitic motion in $\mathrm{x}$-direction eliminated for the desired y-direction motion. 


\section{References}

1. S. Awatar, "Synthesis and analysis of parallel kinematic xy flexure mechanism," $\mathrm{PhD}$ Thesis, Massachusetts Institute of Technology, Cambridge, MA, February 2004.

2. S. Dejima, W. Gao, H. Shimizu, S. Kiyono, and Y. Tomita, "Precision positioning of a five degree-of-freedom planar motion stage," Mechatronics, vol. 15, no. 8, pp. 969-987, October 2005.

3. S. Deshmukh and P. S. Gandhi, "Optomechanical scanning systems for microstereolithography (MSL): Analysis and experimental verification," Journal of Materials Processing Technology, vol. 209, no. 3, pp. 1275-1285, Feburary 2009.

4. S, Awtar, A, Slocum, "Design of parallel kinematic XY flexural mechanism," Proceedings of IDETC/CIE ASME 2005 International Design Engineering Technical Conferences and Computers and Information in Engineering Conference, Long Beach, California, USA, September 2005.

5. P. S. Gandhi, K. Sonawale, and V. Soni, "Development of double parallelogram flexure mechanism via assembly route," 15 th National Conference on Machines and Mechanisms, pp. $1-9,2011$

6. Lobontiu,N, Compliant Mechanisms: Design of Flexure Hinges, CRC Press, 2003.

7. S, Deshmukh, P, Gandhi, "A Novel Optomechatronic Focused Laser Spot Submicron Scanning System for Microstereolithography," Poster presented at International Conference on Nanotechnology, Banglore, India, 2007.

8. Q. Yao, J. Dong, and P. M. Ferreira, "Design, analysis, fabrication and testing of a parallelkinematic micropositioning XY stage," International Journal of Machine Tools and Manufacture, vol. 47, no. 6, pp. 946-961, May 2007.

9. H. S. Kim and Y. M. Cho, "Design and modeling of a novel 3-DOF precision micro-stage," Mechatronics, vol. 19, no. 5, pp. 598-608, August 2009.

10. Y. $\mathrm{Li}$ and Q. Xu, "Modeling and performance evaluation of a flexure-based XY parallel micromanipulator," Mechanism and Machine Theory, vol. 44, no. 12, pp. 2127-2152, December 2009.

11. S. P. Deshmukh, H. Zambare, K. Mate, M. S. Shewale, and Z. Khan, "System identification and PID implementation on double flexural manipulator," 2015 International Conference on Nascent Technologies in the Engineering Field, ICNTE 2015 - Proceedings, pp. 1-5, Feburary 2015.

12. R. Patil, S. Deshmukh, Y. P. Reddy, and K. Mate, "FEA analysis and experimental investigation of building blocks for flexural mechanism," 2015 International Conference on Nascent Technologies in the Engineering Field, ICNTE 2015 - Proceedings, Feburary 2015.

13. Y. Tian, B. Shirinzadeh, D. Zhang, X. Liu, and D. Chetwynd, "Design and forward kinematics of the compliant micro-manipulator with lever mechanisms," Precision Engineering, vol. 33, no. 4, pp. 466-475, October 2009.

14. R. Clement, J. L. Huang, Z. H. Sun, J. Z. Wang, and W. J. Zhang, "Motion and stress analysis of direct-driven compliant mechanisms with general-purpose finite element software," International Journal of Advanced Manufacturing Technology, vol. 65, no. 9-12, pp. 1409-1421, April 2013.

15. S. Lescano et al., "Micromechanisms for Laser Phonosurgery: A Review of Actuators and Compliants Parts . HAL Id : hal-00799755, March 2013.

16. S. Buice, D. Otten, R. H. Yang, S. T. Smith, R. J. Hocken, and D. L. Trumper, "Design evaluation of a single-axis precision controlled positioning stage," Precision Engineering, vol. 33, no. 4, pp. 418-424, October 2009.

17. Y. T. Liu and B. J. Li, "Precision positioning device using the combined piezo-VCM actuator with frictional constraint," Precision Engineering, vol. 34, no. 3, pp. 534-545, July 2010.

18. W. jong Kim, S. Verma, and H. Shakir, "Design and precision construction of novel 
magnetic-levitation-based multi-axis nanoscale positioning systems," Precision Engineering, vol. 31, no. 4, pp. 337-350, October 2007.

19. L. Chu and S. H. Fan, "A novel long-travel piezoelectric-driven linear nanopositioning stage," Precision Engineering, vol. 30, no. 1, pp. 85-95, January 2006.

20. Zhang, C. Chang, T. Ono, and M. Esashi, "A piezodriven XY-microstage for multiprobe nanorecording," Sensors and Actuators, A: Physical, vol. 108, no. 1-3, pp. 230-233, Novermber 2003.

21. H. Liu, W. Y. Jywe, Y. R. Jeng, T. H. Hsu, and Y. tsung Li, "Design and control of a longtraveling nano-positioning stage," Precision Engineering, vol. 34, no. 3, pp. 497-506, July 2010.

22. L. He, H. Fu, D. Sun, M. Karkee, and Q. Zhang, "Shake-and-Catch Harvesting for Fresh Market Apples in Trellis-Trained Trees," Transactions of the ASABE, vol. 60, no. 2, pp. 353-360, 2017.

23. S. Mulik, A. Krishnmoorthy, S. Deshmukh, "Flexural Mechanisms for High Precise Scanning Applications: A Review," International Journal of Mechanical Engineering and Technology, vol. 9, no. 4, pp. 312-327, April 2018.

24. U. Bhagat et al., "Design and analysis of a novel flexure-based 3-DOF mechanism," Mechanism and Machine Theory, vol. 74, pp. 173-187, April 2014.

25. M. S. Shewale et al., "Design and Experimental Validation of Voice Coil Motor for High Precision Applications," presented at the 3rd IEEE International Conference for Convergence in Technology, pp. 1-6, April 6-7, 2018.

26. M. S. Shewale et al., "Design and Implementation of Position Estimator Algorithm on Voice Coil Motor," presented at the 3rd IEEE International Conference for Convergence in Technology pp. 0-4, April 6-7, 2018.

27. S. S. Mulik, S. P. Deshmukh, M. S. Shewale, H. B. Zambare and A. P. Sundare, "Design and implementation of position estimator algorithm on double flexural manipulator," 2017 International Conference on Nascent Technologies in Engineering (ICNTE), Navi Mumbai, 2017, pp. 1-5. doi: 10.1109/ICNTE.2017.7947904 